CERN-TH/96-324

Imperial/TP/96-97/07

hep-th/9611111

\title{
On the structure of composite black p-brane configurations and related black holes
}

\author{
A.A. Tseytlin ${ }^{\star \dagger}$ \\ Theory Division, CERN, CH-1211 Geneve 23, Switzerland \\ and \\ Blackett Laboratory, Imperial College, London, SW7 2BZ, U.K.
}

\begin{abstract}
We comment on the structure of intersecting black p-brane solutions in string theory explaining how known solutions can be obtained from Schwarzschild solution simply by sequences of boosts and dualities. This implies, in particular, that dimensional reduction in all internal world-volume directions including time leads to a metric (related by analytic continuation to a cosmological metric) which does not depend on p-brane charges, i.e. is the same as the metric following by reduction from a higher-dimensional 'neutral' Schwarzschild black hole.
\end{abstract}

November 1996

* e-mail address: tseytlin@ic.ac.uk

$\dagger$ On leave from Lebedev Physics Institute, Moscow. 
Suppose one starts with a configuration of intersecting black p-branes in $D=11$ supergravity, or the corresponding configuration of R-R and NS-NS p-branes in $D=10$ theory, such that it becomes BPS saturated in the extremal limit [1, 2, 3,, 4 . Let the total number of internal directions of intersecting branes (or the dimension of the corresponding anisotropic brane [5]) be $p$ so that the number of remaining 'transverse' spatial and time directions is $D$ (with $D+p=11$ or 10 ). Wrapping the branes around an internal p-torus and dimensionally reducing one finds the following spherically symmetric static black hole (Einstein-frame) metric in $D$ dimensions [6, [7] 1

$$
\begin{gathered}
d s_{D}^{2}=h^{\frac{1}{D-2}}(r)\left[-h^{-1}(r) f(r) d t^{2}+f^{-1}(r) d r^{2}+r^{2} d \Omega_{D-2}^{2}\right], \\
h(r)=\prod_{i=1}^{N} H_{i}(r), \quad f(r)=1-\frac{2 \mu}{r^{D-3}}, \quad H_{i}(r)=1+\frac{\mathcal{Q}_{i}}{r^{D-3}},
\end{gathered}
$$

where $\mathcal{Q}_{i}=\sqrt{Q_{i}^{2}+\mu^{2}}-\mu$ depend on the brane charge parameters $Q_{i}$ and single nonextremality parameter $\mu$. $f$ is the standard Schwarzschild function and $h$ is the product of 'harmonic' functions, 2 one for each brane in the intersection (counting also the linear momentum of a possible boost along a common string as a separate 'charge').

Since this black hole is static, we can formally reduce further in time direction, down to $D-1$ dimensions. This reduction may be motivated by considering a 'cosmological' solution (as, e.g., in [13,14,15]) related to the background (11) by the analytic continuation $t=i y_{0}$, $r=i \tau$ with compact $y_{0} .3$ Then $\int d^{D} x \sqrt{g_{D}} R_{D}+\ldots \rightarrow \int d^{D-1} x V(x) \sqrt{g_{D-1}} R_{D-1}+\ldots, \quad V=$ $\left(h^{\frac{3-D}{D-2}} f\right)^{1 / 2}$, so that transforming to the Einstein frame, $g_{D-1}=V^{\frac{2}{D-3}} g_{D-1}^{(E)}$, one finishes with

$$
d s_{D-1}^{2}=f^{\frac{1}{D-3}}(r)\left[f^{-1}(r) d r^{2}+r^{2} d \Omega_{D-2}^{2}\right] .
$$

This is exactly the same metric that follows simply from the Schwarzschild metric in the space with $p$ isometries, i.e. from the trivial 'neutral black $p$-brane',

$$
d s_{D+p}^{2}=-f(r) d t^{2}+d y_{1}^{2}+\ldots+d y_{p}^{2}+f^{-1}(r) d r^{2}+r^{2} d \Omega_{D-2}^{2}
$$

1 Some special cases were discussed, e.g., in [8, [, 9, 10, 11, 12] (see [6] for a more complete list of references).

2 Non-extremal solutions do not have, of course, static multicenter generalisations.

3 In the case when $D$ is even so that the power of $r$ in $H_{i}$ is odd one needs to rotate also $\mathcal{Q}_{i}$ to get a real metric. Other background fields (i.e. the 3 -tensor if the continuation is done directly at the level of p-brane configuration in $D=11$ ) also remain real.

4 Notice the similarity between $f$-dependence of this metric and $h$-dependence of (1) dimensionally reduced to $D-1$ dimensions along a spatial direction (by taking a periodic array of black holes, etc.). The latter metric turns out to be again (1) with $D$ replaced by $D-1$. 
after one reduces it along all the $p+1$ isometric (p-brane world-volume) directions. The structure of (11) is such that upon reduction in the time direction the dependence on the function $h$, i.e. on the charges of the branes, completely disappears!

In the special case of $D=5$, which is of interest in connection with cosmology in four dimensions, an equivalent observation was recently made in 15.5 This implies that (3) or related cosmological metric can be embedded into any of higher-dimensional black p-brane configurations which correspond to (11).

Our aim below will be to give a simple explanation for this universal decoupling of the brane charges upon reduction in all world-volume directions. In the process, we shall review and clarify the general structure of the composite black brane configurations.

The main message is that the Schwarzschild or the 'neutral' black p-brane solution (3) is, essentially, the 'back-bone' of all such intersecting black p-brane backgrounds in $D=10$ or $D=11$. All $D=11$ solutions in [6] and some of their generalisations [16] can be constructed from (3) with $p \geq 2$ by applying combinations of the following transformations: (a) a Lorentz boost of (3) along one of the isometric directions $y_{i}$; (b) a transformation 'trading' off-diagonal components of the metric for the antisymmetric 3-tensor background, which is just the standard $T$-duality from the point of view of the reduced 10-dimensional theory6; (c) a linear coordinate transformation 'mixing' two of the spatial isometric coordinates, which corresponds to an $S L(2)$ transformation of type IIB 10-dimensional theory 18,17 .

We shall use the familiar $D=10$ supergravity (string-theory) language since the discussion of $D=11$ solutions with $p \geq 2$ isometries can be also rephrased in it. The first two basic generating transformations correspond to $O(p+1, p+1)$ duality. The central starting point is the Schwarzschild metric (3) boosted [19] to some finite momentum along, e.g., $y_{1}$ direction,

$$
\begin{gathered}
d s^{2}=-f(r) d t^{\prime 2}+d y_{1}^{\prime 2}+d y_{2}^{2}+\ldots+d y_{p}^{2}+f^{-1}(r) d r^{2}+r^{2} d \Omega_{D-2}^{2} \\
=-d t^{2}+d y_{1}^{2}+\ldots+d y_{p}^{2}+(f(r)-1)\left(\cosh \beta d t-\sinh \beta d y_{1}\right)^{2}+f^{-1}(r) d r^{2}+r^{2} d \Omega_{D-2}^{2}
\end{gathered}
$$

where

$$
t^{\prime}=\cosh \beta t-\sinh \beta y_{1}, \quad y_{1}^{\prime}=-\sinh \beta t+\cosh \beta y_{1} .
$$

5 See also [14] where the case of the reduction of a single 5-brane was discussed.

6 In the case of at least two spatial isometries the $D=11$ action has the same $T$-duality symmetry as the $D=10$ action in the case of at least one spatial isometry (we are ignoring the Kaluza-Klein modes, i.e. assume that one deals essentially with $D \leq 8$ dimensionally reduced actions [17]). We assume that the time direction is always an isometry, i.e. the total number of isometries in $D=11$ is $\geq 3$. 
The background (四) becomes a gravitational wave in the extremal limit $\mu \rightarrow 0, \beta \rightarrow \infty$, $Q=\mu \sinh 2 \beta=$ fixed. $T$-duality applied in $y_{1}$ direction then leads [20] to a new solution (now already with non-trivial dilaton and $B_{\mu \nu}$ ) - the non-extremal generalisation [\&] of the fundamental string [21], or charged black string. 0 The $S L(2)$ duality of type IIB theory then produces the black R-R string solution. Further $T$-dualities [17] generate various black R-R p-brane backgrounds [8], in particular, the 5-brane of type IIB theory. Applying the $S L(2)$ duality then leads to the solitonic NS-NS 5-brane, thus effectively performing the 'electro-magnetic' duality transformation between the fundamental string and the solitonic 5-brane [9]. The $D=10$ black 2-brane and 5-brane backgrounds can then be lifted to $D=11$, thus explaining the structure of the resulting non-extremal $D=11$ solutions [5] in terms of (4) and lower-dimensional dualities.

To obtain the explicit form of all single black brane backgrounds one, therefore, never needs to solve the supergravity equations of motion: the only input is the Schwarzschild solution (3) (in its coordinate-transformed form (田) and $T$ and $S L(2)$ symmetries of the supergravity actions (or $U$-duality symmetry of the $D=8$ action [17]). To construct all extremal solutions it is sufficient to start with the 0-brane background (with some number $p$ of additional isometries) and apply $O(p+1, p+1)$ and $S L(2)$ dualities.

Having found a single black brane background, one may then repeat the procedure, by using it, instead of (ब1), as a starting point. Appropriately boosting and applying the duality transformations (and 'smearing' when necessary in internal directions) one can then generate backgrounds describing intersections of pairs of branes. 6 Each 'boost+duality' cycle introduces a new charge or function $H_{i}$ into the intersecting brane solution (and thus into the corresponding black hole metric (1)). This essentially explains the applicability of the 'harmonic function rule' [2] (or its non-extremal version [6]) to the construction of such backgrounds.

Let us illustrate this construction more explicitly on some basic examples of backgrounds corresponding to threshold and non-threshold bound states of branes. Having found the charged black string by T-duality from boosted Schwarzschild (neutral string) background, we can finitely boost it in the longitudinal direction (the string metric is not boost-invariant in the non-extremal case). The resulting solution is parametrised by two charges (two harmonic functions in the extremal limit) and represents a wound string with a linear momentum flow along it [23,24], or it can be viewed as a combination of a fundamental string and a wave, $1_{N S}+\uparrow$. All other possible (threshold or non-threshold)

7 More precisely, for $p>1$ we get a string 'smeared' in other $p-1$ isometric directions, or, in extremal limit, as a periodic array of strings. Equivalently, this background can be interpreted as a black p-brane with a charge along $y_{1}$ string direction only.

8 For pairs of intersecting R-R branes this can be done simply by making formal $T$-duality transformations as was demonstrated in the extremal case in [22]. 
configurations of intersecting branes with two charges can now be obtained from $1_{N S}+\uparrow$ by various sequences of dualities. For example, $S L(2)$ leads to R-R string with momentum, $1_{R}+\uparrow$, and then $T$-duality in the orthogonal isometric directions produces other longitudinally boosted p-brane backgrounds, e.g., $4+\uparrow, 5_{R}+\uparrow$ and $6+\uparrow$. T-duality in the direction of the momentum flow then gives $3 \perp 1_{N S}, 4 \perp 1_{N S}$, and $5_{R} \perp 1_{N S}$. SL(2) transforms $5_{R}+\uparrow$ into $5_{N S}+\uparrow$, leading, after $T$-duality, to the explicit form of non-extremal solitonic 5 -brane plus fundamental string $5_{N S}+1_{N S}$ background (and, further, to $5_{R}+1_{R}$ after $S L(2)$ duality, $4+0$ after $T$-duality, etc.). Alternatively, applying $S L(2)$ rotation to the black string background one finds the non-extremal version of the string-string bound state, $1_{N S}+1_{R}$ [18]. Other non-threshold bound state configurations with two charges are related to $1_{N S}+1_{R}$ by the dualities: $T$-duality gives $3+1_{N S}, S L(2)$ transforms this into $3+1_{R}, T$-duality along the string direction produces $2+0$ configuration, etc. The explicit form of these backgrounds was given in [16].

To add a third charge (a third brane in the intersection) one is to boost the twobrane configuration. For example, boosting $5_{N S}+1_{N S}$ along the string direction we obtain $5_{N S}+1_{N S}+\uparrow$ and thus also the dual backgrounds $5_{R}+1_{R}+\uparrow, 4+0 \perp 1$, etc. Compactification then leads to the three-charge $D=5$ black hole 9

Notice that one never needs to decide which combinations of branes are possible - they are automatically selected by the duality transformations. 10 As the duality transformations preserve supersymmetry of the extremal solutions, one may say that the origin of the $1 / 2$ supersymmetry of the single branes (or non-threshold bound states related to some of them by $S L(2)$ rotations) is in supersymmetry of a wave or $T$-dual fundamental string background, while the reason for the $1 / 4$ supersymmetry of the threshold combinations of two branes is in the $1 / 4$ supersymmetry of the fundamental string with momentum, etc. Another remark is that the procedure described above implies that the resulting non-extremal solutions have just one non-extremality parameter - the mass of the original Schwarschild solution, i.e. they are one-parameter 'deformations' of the supersymmetric BPS backgrounds.

Now, why the above construction guarantees that if one reduces in all internal brane directions and time direction one should get the metric (2) with no trace of the $H_{i}$-functions? There are two simple reasons. First reason is that if one reduces also in time direction (or in its compact analytic continuation) the boost (5) becomes simply an 'internal' coordinate

9 This construction of the non-extremal version of the black hole corresponding to $5_{N S}+1_{N S}+\uparrow$ solution of [25] is different from the one in [12] in that here the starting point is not the equalcharge Reissner-Nordström type solution but just the Schwarzschild solution (or neutral 5-brane) and all three charges are added by boosts interchanged with duality transformations.

10 This is true not only for combinations of R-R branes where there is a microscopic D-brane explanation for T-duality relations [26] but also for 'mixed' NS-NS and R-R combinations. 
transformation which does not influence the dimensionally reduced metric. Second is the duality invariance of the reduced Einstein-frame metric.

This explanation of why the intersecting brane metric or (11) always reduces to (2) may have some implications for an interesting recent discussion 115 of possible stringtheory resolution of cosmological singularities. As far as the Einstein-frame metric is concerned, all 'exotic' higher-dimensional embeddings of (2) which are related to (R-R, NS-NS, or 'mixed') p-brane configurations should be considered on an equal footing with the simplest 'neutral' Schwarzschild embedding (3). It remains to be seen whether the presence of additional matter fields makes some of more complicated embeddings more promising. 10

This work was partially supported by PPARC, the European Commission TMR programme ERBFMRX-CT96-0045 and NATO grant CRG 940870.

11 In particular, there is a question of whether $D$-branes should have any special importance in this context (given also that the non-extremality of the solutions is essential here: for $\mu=0$ (2) becomes simply a flat space). 


\section{References}

[1] G. Papadopoulos and P.K. Townsend, Phys. Lett. B380 (1996) 273, hep-th/9603087.

[2] A.A. Tseytlin, Nucl. Phys. B475 (1996) 149, hep-th/9604035.

[3] I.R. Klebanov and A.A. Tseytlin, Nucl. Phys. B475 (1996) 179, hep-th/9604166.

[4] J.P. Gauntlett, D.A. Kastor and J. Traschen, hep-th/9604179.

[5] R. Güven, Phys. Lett. B276 (1992) 49.

[6] M. Cvetič and A.A. Tseytlin, Nucl. Phys. B478 (1996) 181, hep-th/9606033.

[7] I.R. Klebanov and A.A. Tseytlin, Nucl. Phys. B475 (1996) 179, hep-th/9607107.

[8] G.T. Horowitz and A. Strominger, Nucl. Phys. B360 (1991) 197.

[9] M.J. Duff, R.R. Khuri and J.X. Lu, Phys. Rept. 259 (1995) 213, hep-th/9412184.

[10] M.J. Duff, H. Lü and C.N. Pope, Phys. Lett. B382 (1996) 73, hep-th/960405.

[11] M. Cvetič and D. Youm, Nucl. Phys. B476 (1996) 118, hep-th/9603100.

[12] G. Horowitz, J. Maldacena and A. Strominger, Phys. Lett. B383 (1996) 151, hepth/9603109.

[13] K. Behrndt and S. Förste, Nucl. Phys. B430 (1994) 441, hep-th/9403179.

[14] R. Poppe and S. Schwager, hep-th/9610166.

[15] F. Larsen and F. Wilczek, hep-th/9610252.

[16] J.G. Russo and A.A. Tseytlin, hep-th/9611047.

[17] E. Bergshoeff, C. Hull and T. Ortin, Nucl. Phys. B451 (1995) 547, hep-th/9504081.

[18] J.H. Schwarz, Phys. Lett. B360 (1995) 13, hep-th/9508143.

[19] G.W. Gibbons, Nucl. Phys. B207 (1982) 337.

[20] J.H. Horne, G.T. Horowitz and A.R. Steif, Phys. Rev. Lett. 68 (1992) 568; G. Horowitz, in String Theory and Quantum Gravity '92, ed. by J. Harvey et al. (World Scientific, Singapore, 1993).

[21] A. Dabholkar, G.W. Gibbons, J. Harvey and F. Ruiz Ruiz, Nucl. Phys. B340 (1990) 33.

[22] K. Behrndt, E. Bergshoeff and B. Janssen, hep-th/9604168.

[23] D. Waldram, Phys. Rev. D47 (1993) 2528.

[24] G.T. Horowitz and A.A. Tseytlin, Phys. Rev. D51 (1995) 2896, hep-th/9409021.

[25] A.A. Tseytlin, Mod. Phys. Lett. A11 (1996) 689, hep-th/9601177.

[26] J. Polchinski, S. Chaudhuri and C.V. Johnson, hep-th/9602052. 